Article

\title{
Bio-Guided Isolation of the Cytotoxic Terpenoids from the Roots of Euphorbia kansui against Human Normal Cell Lines L-O2 and GES-1
}

\section{Li Zhang, Lan Gao, Zhengjun Li, Xiaojing Yan, Yanjing Yang, Yuping Tang *, Yudan Cao and Anwei Ding *}

Jiangsu Key Laboratory for High Technology Research of TCM Formulae, Nanjing University of Chinese Medicine, Nanjing 210046, China; E-Mails: zhangli@njutcm.edu.cn (L.Z.); gaolan1000@126.com (L.G.); lizhengjun404@126.com (Z.L.); yanxiaojing963@163.com (X.Y.); candy-jing@qq.com (Y.Y.); raindc@163.com (Y.C.)

* Authors to whom correspondence should be addressed; E-Mails: yupingtang@njutcm.edu.cn(Y.T.); awding105@163.com (A.D.); Tel./Fax: +86-25-8581-1916 (Y.T.); +86-25-8581-1523(A.D.).

Received: 26 July 2012; in revised form: 27 August 2012 / Accepted: 29 August 2012 /

Published: 10 September 2012

\begin{abstract}
The dried roots of Euphorbia kansui (kansui) have been used for centuries in China as a herbal medicine for edema, ascites, and asthma. The 95\% ethanol extract showed a significant inhibition of cell proliferation against human normal cell lines L-O2 and GES-1. Bioassay-guided separation of the 95\% ethanol extract from the roots of E. kansui led to the isolation of 12 diverse terpenoids whose structures were identified by ${ }^{1} \mathrm{H}$, ${ }^{13} \mathrm{C}$ NMR spectroscopy and ESI-MS as kansuinine A (1), kansuinine B (2), kansuinine C (3), kansuiphorin $\mathrm{C}$ (4), 3-O-(2'E,4'Z-decadienoyl)-20-O-acetylingenol (5), 3-O-(2'E,4'Edecadienoyl)-20-O-acetylingenol (6), 3-O-(2'E,4'Z-decadienoyl)-20-deoxyingenol (7), 3-O-benzoyl-20-deoxyingenol (8), 5-O-benzoyl-20-deoxyingenol (9), kansenone (10), epi-kansenone (11), euphol (12). All these 12 terpernoids were evaluated in vitro for cytotoxicity on L-O2 and GES-1 cell lines. Most ingenane-type diterpenoids and 8-ene-7-one triterpenoids (5-11) exhibited a relatively lower $\mathrm{IC}_{50}$ value; therefore, these compounds had stronger cytotoxicity against human normal cell lines L-O2 and GES-1 with dose-dependent relationships. These results will be significantly helpful to reveal the mechanism of toxicity of kansui and to effectively guide safer clinical application of this herb.
\end{abstract}


Keywords: Euphorbia kansui; terpenoids; cytotoxicity; bio-guided separation; human normal liver cell lines L-O2; human normal gastric epithelial cell line GES-1

\section{Introduction}

With the development of science and technology, and the increasing health awareness and life expectancy of humans, the safety problem of traditional Chinese medicines (TCM) is attracting more and more attention. The dried roots of Euphorbia kansui T.N. Liou ex T.P. Wang, known as "kansui," which were recorded in Shennong-Bencao and have been used for centuries in China as a herbal medicine for edema, ascites, and asthma [1,2]. Recently, it was found that kansui has excellent prospects for the treatment of cancer [2-6], pancreatitis [7-9], and intestinal obstruction [10,11]. However, kansui causes severe skin, oral, and gastrointestinal irritation, hepatic injury and tumor promoting toxicity, which have seriously restricted its clinical application [12-16]. So, outweighing all other considerations is the need to understand the toxicity mechanism of kansui, and further to reduce its toxicity without affecting its therapeutic action.

Previous phytochemical investigation resulted in several terpenoids and phenolic derivatives isolated and identified from kansui [13,17-23]. It was reported that the terpenoids in kansui, including jatrophane-type diterpenoids, ingenane-type diterpenoids and triterpenoids, showed comprehensive bio-activities such as the anti-leukemia [2], anti-tumor [2-5], anti-allergy [24], anti-virus [13,25], and antinematodal activity [26]. On the other hand, some of the terpenoids had a serious proinflammatory effect $[27,28]$. Our recent study also discovered that kansui exhibited serious gastrointestinal toxicity and hepatotoxicity [14-16,29]. In the present study, the cytotoxicity of kansui extracts was evaluated in vitro against human normal cell lines L-O2 and GES-1. The 95\% ethanol extract showed a significant inhibition of cell proliferation against two human normal cell lines. Bioassay-guided isolation of pure compounds was carried out, their structures were identified, and their structure-activity relationships were further studied.

\section{Results and Discussion}

\subsection{Identification of Compounds 1-12}

On the basis of cytotoxicity assay results of different extracts and pure compounds against human normal cell lines L-O2 and GES-1 (Table 4), 12 terpenoids were isolated from the EtOAc extract of kansui. By spectral and physiochemical data analysis and/or comparison with literatures data, their structures were elucidated as kansuinines A, B, C (1-3) [17,21], kansuiphorin C (4) [20], 3-O-(2'E, 4'Zdecadienoyl)-20-O-acetylingenol (5) [17], 3-O-(2'E,4'E-decadienoyl)-20-O-acetylingenol (6) [17], 3-O-(2'E,4'Z-decadienoyl)-20-deoxyingenol (7) [17,23], 3-O-benzoyl-20-deoxyingenol (8) [22], 5-O-benzoyl-20-deoxyingenol (9) [17], kansenone (10) [17], epi-kansenone (11) [17], and euphol (12) [17,21] (Figure 1). This was the first report of the bio-guided isolation of different fractions and pure terpenoids from kansui extracts by the gastrointestinal and hepatic cytotoxicity assay against human normal cell lines L-O2 and GES-1. 
Figure 1. The chemical structures of compounds 1-12.

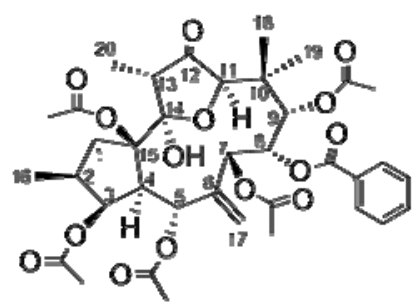

1

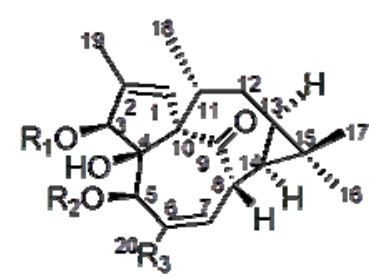

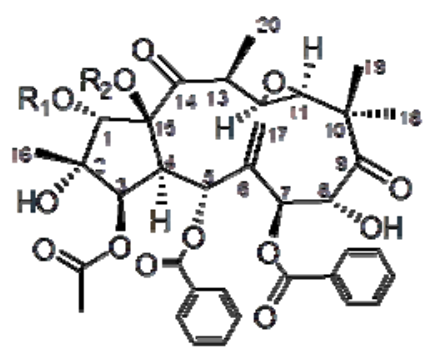

$4 \mathrm{COCH}_{3}$

$E R$

$5 \mathrm{CO}(\mathrm{CH}=\mathrm{CH})_{2}\left(\mathrm{CH}_{2}\right)_{2} \mathrm{CH}_{3} \quad \mathrm{H}$

$E Z$

$6 \mathrm{CO}(\mathrm{CH}=\mathrm{CH})_{2}\left(\mathrm{CH}_{2}\right)_{4} \mathrm{CH}_{3} \quad \mathrm{H}$

$E R$

$7 \mathrm{CO}(\mathrm{CH}=\mathrm{CH})_{2}\left(\mathrm{CH}_{2}\right)_{4} \mathrm{CH}_{3} \quad \mathrm{H}$

$\mathrm{COC}_{6} \mathrm{H}_{\mathrm{s}}$

H
$\mathbf{R}_{\text {. }} \quad \mathbf{R}_{\text {子 }}$

$\mathrm{COC}_{2} \mathrm{H}_{3} \quad \mathrm{CH}_{3}$

$2 \mathbf{R}_{\mathrm{I}}=\mathrm{COCH}_{3} \mathbf{R}_{2}=\mathrm{H}$

$3 \mathbf{R}_{\mathrm{I}}=\mathbf{H} \quad \mathbf{R}_{2}=\mathrm{COCH}_{2}$

$\mathrm{CH}_{2} \mathrm{OCOCH}$

$\mathrm{CH}_{2} \mathrm{OCOCH}$

$\mathrm{CH}_{3}$

$\mathrm{CH}_{3}$

$\mathrm{COC}_{2} \mathrm{H}_{3} \quad \mathrm{CH}_{3}$

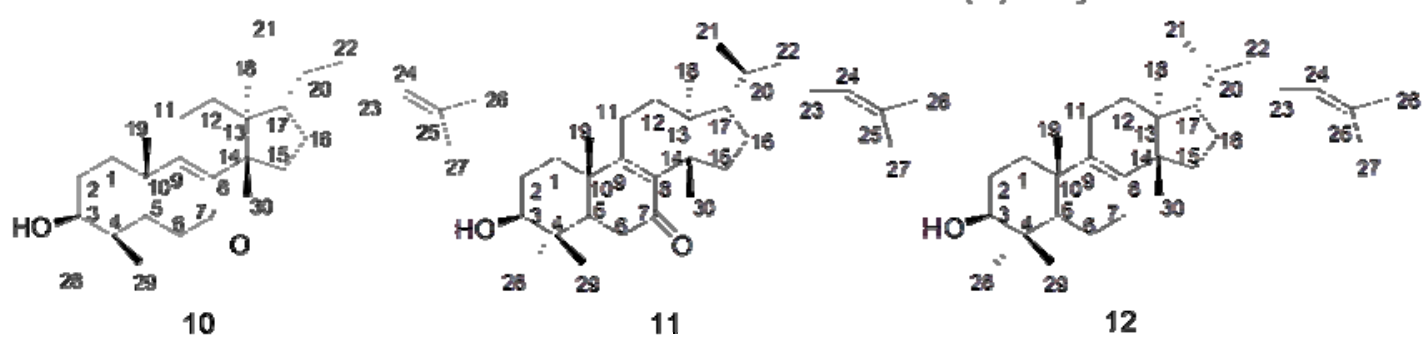

Kansuinine A (1): white powder, mp 218-220 ${ }^{\circ} \mathrm{C} ; R_{\mathrm{f}}=0.25\left(\mathrm{SiO}_{2}\right.$, petroleum ether-chloroformEtOAc $=5: 3: 3)$; UV $(\mathrm{MeOH}) \lambda_{\max } 232 \mathrm{~nm}$; ESI-MS (positive) $m / z 753[\mathrm{M}+\mathrm{Na}]^{+} ;{ }^{1} \mathrm{H}-\mathrm{NMR}$ data, see Table 1.

Kansuinine B (2): colorless crystal; $\mathrm{mp} 215-216^{\circ} \mathrm{C} ; R_{\mathrm{f}}=0.30\left(\mathrm{SiO}_{2}\right.$, petroleum ether-chloroformEtOAc $=5: 3: 3$ ); UV $(\mathrm{MeOH}) \lambda_{\max } 232 \mathrm{~nm}$; ESI-MS (positive) $m / z 745[\mathrm{M}+\mathrm{Na}]^{+}$; ${ }^{1} \mathrm{H}-\mathrm{NMR}$ data, see Table 1.

Kansuinine C (3): colorless needle crystal; mp 217-218 ${ }^{\circ} \mathrm{C} ; R_{\mathrm{f}}=0.28\left(\mathrm{SiO}_{2}\right.$, petroleum ether-chloroform-EtOAc $=5: 3: 3$ ); UV $(\mathrm{MeOH}) \lambda_{\max } 232 \mathrm{~nm}$; ESI-MS (positive) $\mathrm{m} / z 745[\mathrm{M}+\mathrm{Na}]^{+}$; ${ }^{1} \mathrm{H}-\mathrm{NMR}$ data, see Table 1.

Kansuiphorin C (4): colorless gum; $R_{\mathrm{f}}=0.83\left(\mathrm{SiO}_{2}\right.$, petroleum ether-chloroform-EtOAc $\left.=5: 3: 1\right)$; $\mathrm{UV}(\mathrm{MeOH}) \lambda_{\max } 230$; ESI-MS (positive) $\mathrm{m} / z 501[\mathrm{M}+\mathrm{Na}]^{+} ;{ }^{1} \mathrm{H}-\mathrm{NMR}$ data, see Table 2.

3-O-(2'E,4'Z-Decadienoyl)-20- $O$-acetylingenol (5): colorless gum; $R_{\mathrm{f}}=0.55\left(\mathrm{SiO}_{2}\right.$, petroleum ether-chloroform-EtOAc $=5: 3: 2)$; UV $(\mathrm{MeOH}) \lambda_{\max } 266$, 206; ESI-MS (positive) $m / z 563[\mathrm{M}+\mathrm{Na}]^{+}$; ${ }^{1} \mathrm{H}-\mathrm{NMR}$ data, see Table 2.

3-O-(2'E,4'E-Decadienoyl)-20-O-acetylingenol (6): colorless gum; $R_{\mathrm{f}}=0.65\left(\mathrm{SiO}_{2}\right.$, petroleum ether-chloroform-EtOAc $=5: 3: 2$ ); UV $(\mathrm{MeOH}) \lambda_{\max } 266$, 203; ESI-MS (positive) $m / z 563[\mathrm{M}+\mathrm{Na}]^{+}$; ${ }^{1} \mathrm{H}-\mathrm{NMR}$ data, see Table 2. 
Table 1. The ${ }^{1} \mathrm{H}-\mathrm{NMR}$ spectral assignments ( $\left.\delta / \mathrm{ppm}\right)$ for compounds $\mathbf{1}-\mathbf{3}$ in $\mathrm{CDCl}_{3}(300 \mathrm{MHz})$.

\begin{tabular}{|c|c|c|c|c|}
\hline Position & 1 & Position & 2 & 3 \\
\hline \multirow[t]{2}{*}{1} & $2.66(\mathrm{dd}, 6.3,14.1)$ & 1 & 4.33 (brs) & $4.94(\mathrm{~s})$ \\
\hline & $2.25(\mathrm{~m})$ & & & \\
\hline 2 & $2.10(\mathrm{~m})$ & 2 & - & - \\
\hline 3 & 5.60 (brs) & 3 & $5.53(\mathrm{~d}, 4.8)$ & $5.38(\mathrm{~d}, 4.7)$ \\
\hline 4 & 2.98 (brs) & 4 & $3.63(\mathrm{dd}, 11.4,5.0)$ & $3.48(\mathrm{~m})$ \\
\hline 5 & $6.15(\mathrm{~s})$ & 5 & $5.93(\mathrm{~d}, 5.85)$ & $5.95(\mathrm{~s})$ \\
\hline 7 & $6.44(\mathrm{~s})$ & 7 & $5.88(\mathrm{~s})$ & $5.88(\mathrm{~s})$ \\
\hline 8 & $6.05(\mathrm{~s})$ & 8 & 4.66 (brs) & $4.70(\mathrm{~d}, 9.3)$ \\
\hline 9 & $5.09(\mathrm{~s})$ & 9 & - & - \\
\hline 11 & $4.14(\mathrm{~s})$ & 11 & $3.70(\mathrm{~d}, 1.8)$ & $3.65(\mathrm{~d}, 2.04)$ \\
\hline 12 & - & 12 & $3.33(\mathrm{~m})$ & $3.46(\mathrm{~m})$ \\
\hline 13 & $2.33(\mathrm{q}, 6.6)$ & 13 & $3.38(\mathrm{~m})$ & $3.26(\mathrm{~m})$ \\
\hline 16 & $0.936(\mathrm{~d}, 6.6)$ & 16 & $1.29(\mathrm{~s})$ & $1.32(\mathrm{~s})$ \\
\hline \multirow[t]{2}{*}{17} & $5.24(\mathrm{~s})$ & 17 & $6.52(\mathrm{~s})$ & $6.390(\mathrm{~s})$ \\
\hline & $5.14(\mathrm{~s})$ & & $5.94(\mathrm{~s})$ & $5.95(\mathrm{~s})$ \\
\hline 18 & $1.31(\mathrm{~s})$ & 18 & $1.35(\mathrm{~s})$ & $1.33(\mathrm{~s})$ \\
\hline 19 & $1.15(\mathrm{~s})$ & 19 & $0.85(\mathrm{~s})$ & $0.89(\mathrm{~s})$ \\
\hline 20 & $1.32(\mathrm{~d}, 6.3)$ & 20 & $1.53(\mathrm{~d}, 6.27)$ & $1.65(\mathrm{~d}, 6.51)$ \\
\hline Acetyls & - & Acetyls & - & - \\
\hline COMe-1 & - & COMe-1 & - & $2.14(\mathrm{~s})$ \\
\hline COMe-3 & $2.10(\mathrm{~s})$ & COMe-3 & $1.90(\mathrm{~s})$ & $1.95(\mathrm{~s})$ \\
\hline COMe-5 & $2.08(\mathrm{~s})$ & COMe-5 & - & - \\
\hline COMe-7 & $2.19(\mathrm{~s})$ & COMe-7 & - & - \\
\hline COMe-9 & $2.01(\mathrm{~s})$ & COMe-9 & - & - \\
\hline COMe-15 & $1.96(\mathrm{~s})$ & COMe-15 & $2.32(\mathrm{~s})$ & - \\
\hline Benzoyls & - & Benzoyls & - & - \\
\hline COPh- $8-2,6$ & $8.05(\mathrm{~m})$ & COPh-5-2,6 & $7.56(\mathrm{~m})$ & $7.48(\mathrm{~m})$ \\
\hline$-3,5$ & $7.44(\mathrm{~m})$ & $-3,5$ & $7.03(\mathrm{~m})$ & $6.88(\mathrm{~m})$ \\
\hline-4 & $7.55(\mathrm{~m})$ & -4 & $7.22(\mathrm{~m})$ & $7.06(\mathrm{~m})$ \\
\hline $\mathrm{COPh}-7-2,6$ & - & $\mathrm{COPh}-7-2,6$ & $7.51(\mathrm{~m})$ & $7.53(\mathrm{~m})$ \\
\hline$-3,5$ & - & $-3,5$ & $6.93(\mathrm{~m})$ & $7.02(\mathrm{~m})$ \\
\hline-4 & - & -4 & $7.10(\mathrm{~m})$ & $7.25(\mathrm{~m})$ \\
\hline OH-1 & - & $\mathrm{OH}-1$ & 3.94 (brs) & - \\
\hline $\mathrm{OH}-2$ & - & $\mathrm{OH}-2$ & $2.53(\mathrm{~m})$ & $2.33(\mathrm{~s})$ \\
\hline $\mathrm{OH}-8$ & - & OH-8 & $3.59(\mathrm{~m})$ & $3.50(\mathrm{~m})$ \\
\hline OH-15 & - & $\mathrm{OH}-15$ & - & $4.10(\mathrm{~s})$ \\
\hline
\end{tabular}

3-O-(2'E,4'Z-Decadienoyl)-20-deoxyingenol (7): colorless gum; $R_{\mathrm{f}}=0.66\left(\mathrm{SiO}_{2}\right.$, petroleum ether-chloroform-EtOAc $=5: 3: 2)$; UV $(\mathrm{MeOH}) \lambda_{\max } 260$, 206; ESI-MS (positive) $\mathrm{m} / z 505[\mathrm{M}+\mathrm{Na}]^{+}$; ${ }^{1} \mathrm{H}-\mathrm{NMR}$ data, see Table 2.

3-O-Benzoyl-20-deoxyingenol (8): colorless gum; $R_{\mathrm{f}}=0.72\left(\mathrm{SiO}_{2}\right.$, petroleum ether-chloroformEtOAc $=5: 3: 2$ ); UV $(\mathrm{MeOH}) \lambda_{\max } 273,229,208$; ESI-MS (positive) $m / z 459[\mathrm{M}+\mathrm{Na}]^{+} ;{ }^{1} \mathrm{H}-\mathrm{NMR}$ data, see Table 2. 
Table 2. The ${ }^{1} \mathrm{H}-\mathrm{NMR}$ spectral assignments ( $\left.\delta / \mathrm{ppm}\right)$ for compounds $\mathbf{4}-\mathbf{9}$ in $\mathrm{CDCl}_{3}(300 \mathrm{MHz})$.

\begin{tabular}{|c|c|c|c|c|c|c|}
\hline Position & 4 & 5 & 6 & 7 & 8 & 9 \\
\hline 1 & 6.14 (brs) & $6.05(\mathrm{~s})$ & $6.04(\mathrm{~s})$ & $6.07(\mathrm{~s})$ & $6.14(\mathrm{~s})$ & $6.02(\mathrm{~s})$ \\
\hline 3 & $5.07(\mathrm{~s})$ & $5.60(\mathrm{~s})$ & $5.57(\mathrm{~s})$ & $5.53(\mathrm{~s})$ & $5.67(\mathrm{~s})$ & $3.73(\mathrm{~s})$ \\
\hline 5 & 5.51 (brs) & $3.88(\mathrm{~s})$ & $3.88(\mathrm{~s})$ & $3.68(\mathrm{~s})$ & 3.76 (brs) & $5.41(\mathrm{~s})$ \\
\hline 7 & $\begin{array}{c}5.88 \\
(\mathrm{~d}, 1.5)\end{array}$ & $\begin{array}{c}6.12 \\
(\mathrm{~d}, 3.7)\end{array}$ & $\begin{array}{c}6.12 \\
(d, 3.7)\end{array}$ & $5.76(\mathrm{~m})$ & $5.78(\mathrm{~m})$ & $5.91(\mathrm{~m})$ \\
\hline 8 & $\begin{array}{l}4.26(\mathrm{dd} \\
9.5,1.5)\end{array}$ & $\begin{array}{l}4.08(\mathrm{dd}, \\
4.0,11.7)\end{array}$ & $\begin{array}{l}4.08(\mathrm{dd}, \\
4.0,11.7)\end{array}$ & $\begin{array}{l}4.02(\mathrm{dd}, \\
9.9,3.0)\end{array}$ & $4.03(\mathrm{~m})$ & $\begin{array}{c}4.13(\mathrm{dd}, 7.0 \\
14.2)\end{array}$ \\
\hline 11 & $2.31(\mathrm{~m})$ & $2.49(\mathrm{~m})$ & $2.48(\mathrm{~m})$ & $2.46(\mathrm{~m})$ & $2.54(\mathrm{~m})$ & $2.41(\mathrm{~m})$ \\
\hline 12 & $2.54(\mathrm{~m})$ & $2.26(\mathrm{~m})$ & $2.26(\mathrm{~m})$ & $2.26(\mathrm{~m})$ & $2.28(\mathrm{~m})$ & $2.34(\mathrm{~m}) 1.77$ \\
\hline & $1.73(\mathrm{~m})$ & $1.75(\mathrm{~m})$ & $1.76(\mathrm{~m})$ & $1.74(\mathrm{~m})$ & $1.75(\mathrm{~m})$ & (m) \\
\hline 13 & $0.74(\mathrm{~m})$ & $0.72(\mathrm{~m})$ & $0.72(\mathrm{~m})$ & $0.67(\mathrm{~m})$ & $0.67(\mathrm{~m})$ & $0.70(\mathrm{~m})$ \\
\hline 14 & $0.88(\mathrm{~m})$ & $0.98(\mathrm{~m})$ & $0.98(\mathrm{~m})$ & $0.90(\mathrm{~m})$ & $0.93(\mathrm{~m})$ & $0.94(\mathrm{~m})$ \\
\hline 16 & $1.07(\mathrm{~s})$ & $1.06(\mathrm{~s})$ & $1.06(\mathrm{~s})$ & $1.05(\mathrm{~s})$ & $1.04(\mathrm{~s})$ & $1.07(\mathrm{~s})$ \\
\hline 17 & $1.13(\mathrm{~s})$ & $1.08(\mathrm{~s})$ & $1.08(\mathrm{~s})$ & $1.08(\mathrm{~s})$ & $1.06(\mathrm{~s})$ & $1.17(\mathrm{~s})$ \\
\hline 18 & $\begin{array}{c}1.00 \\
(\mathrm{~d}, 4.7)\end{array}$ & $\begin{array}{c}1.00 \\
(\mathrm{~d}, 7.3)\end{array}$ & $\begin{array}{c}1.00 \\
(\mathrm{~d}, 7.3)\end{array}$ & $\begin{array}{c}0.99 \\
(\mathrm{~d}, 7.2)\end{array}$ & $\begin{array}{c}1.05 \\
(\mathrm{~d}, 4.2)\end{array}$ & $\begin{array}{c}0.98 \\
(\mathrm{~d}, 5.61)\end{array}$ \\
\hline 19 & 1.78 (brs) & $1.8(\mathrm{~d}, 1.1)$ & $1.8 \mathrm{~d}(1.1)$ & 1.80 (brs) & $\begin{array}{c}1.83 \\
(\mathrm{~d}, 1.5)\end{array}$ & $1.83(\mathrm{~s})$ \\
\hline 20 & $1.56(\mathrm{~s})$ & $\begin{array}{c}4.75,4.49 \\
(\mathrm{Abq}, 12.5)\end{array}$ & $\begin{array}{c}4.75,4.49 \\
(\text { Abq, } 12.5)\end{array}$ & $1.79(\mathrm{~s})$ & $1.81(\mathrm{~s})$ & $1.60(\mathrm{~s})$ \\
\hline $3-\mathrm{R}, 2^{\prime}$ & $8.13(\mathrm{~m})$ & $\begin{array}{c}5.95 \\
(\mathrm{~d}, 15.3)\end{array}$ & $\begin{array}{c}5.86 \\
(\mathrm{~d}, 15.3)\end{array}$ & $\begin{array}{c}5.95 \\
(\mathrm{~d}, 5.1)\end{array}$ & $\begin{array}{c}8.04 \\
(\mathrm{~d}, 7.3)\end{array}$ & - \\
\hline $3^{\prime}$ & $7.48(\mathrm{~m})$ & $7.70(\mathrm{~m})$ & $7.31(\mathrm{~m})$ & $\begin{array}{c}7.68(\mathrm{dd}, \\
15.3,11.4)\end{array}$ & $\begin{array}{c}7.48 \\
(t, 7.6)\end{array}$ & - \\
\hline $4^{\prime}$ & $7.60(\mathrm{~m})$ & $6.16(\mathrm{~m})$ & $6.21(\mathrm{~m})$ & $6.16(\mathrm{~m})$ & $\begin{array}{c}7.61 \\
(t, 7.3)\end{array}$ & - \\
\hline $5^{\prime}$ & - & $5.95(\mathrm{~m})$ & $6.21(\mathrm{~m})$ & $5.94(\mathrm{~m})$ & - & - \\
\hline $6^{\prime}$ & - & $2.29(\mathrm{~m})$ & $2.20(\mathrm{~m})$ & $2.34(\mathrm{~m})$ & - & - \\
\hline $7^{\prime}$ & - & $1.43(\mathrm{~m})$ & $1.43(\mathrm{~m})$ & $1.43(\mathrm{~m})$ & - & - \\
\hline $8^{\prime}$ & - & $1.31(\mathrm{~m})$ & $1.31(\mathrm{~m})$ & $120(\mathrm{~m})$ & - & - \\
\hline $9^{\prime}$ & - & $1.28(\mathrm{~m})$ & $1.28(\mathrm{~m})$ & $1.29(\mathrm{~m})$ & - & - \\
\hline $10^{\prime}$ & - & $0.89(\mathrm{t}, 7.0)$ & $\begin{array}{c}0.72 \\
(t, 7.0)\end{array}$ & $0.89(\mathrm{~m})$ & - & - \\
\hline OAc & - & $2.06(\mathrm{~s})$ & $2.05(\mathrm{~s})$ & - & - & - \\
\hline $5-\mathrm{R}, 2^{\prime}$ & $2.05(\mathrm{~s})$ & - & - & - & - & $8.10(\mathrm{~m})$ \\
\hline $3^{\prime}$ & - & - & - & - & - & $7.46(\mathrm{~m})$ \\
\hline $4^{\prime}$ & - & - & - & - & - & $7.60(\mathrm{~m})$ \\
\hline
\end{tabular}

5-O-Benzoyl-20-deoxyingenol (9): colorless gum; $R_{\mathrm{f}}=0.61\left(\mathrm{SiO}_{2}\right.$, petroleum ether-chloroformEtOAc $=5: 3: 2) ; \mathrm{UV}(\mathrm{MeOH}) \lambda_{\max } 273,229,208 ;$ ESI-MS (positive) $m / z 459[\mathrm{M}+\mathrm{Na}]^{+} ;{ }^{1} \mathrm{H}-\mathrm{NMR}$ data, see Table 2.

Kansenone (10): colorless gum; $R_{\mathrm{f}}=0.61\left(\mathrm{SiO}_{2}\right.$, petroleum ether-chloroform-EtOAc $\left.=5: 3: 2\right)$; UV (MeOH) $\lambda_{\max } 252$; ESI-MS (positive) $m / z 441[\mathrm{M}+\mathrm{H}]^{+}$; ${ }^{1} \mathrm{H}-\mathrm{NMR}$ data, see Table 3. 
Table 3. The ${ }^{1} \mathrm{H}-\mathrm{NMR}$ spectral assignments $(\delta / \mathrm{ppm})$ for compounds $\mathbf{1 0}$ and $\mathbf{1 1}$ in $\mathrm{CDCl}_{3}(300 \mathrm{MHz})$.

\begin{tabular}{cccccc}
\hline Position & $\mathbf{1 0}$ & $\mathbf{1 1}$ & Position & $\mathbf{1 0}$ & $\mathbf{1 1}$ \\
\hline $1 \alpha$ & $1.44(\mathrm{~m})$ & $1.44(\mathrm{~m})$ & $16 \beta$ & $1.93(\mathrm{~m})$ & $1.94(\mathrm{~m})$ \\
$1 \beta$ & $1.86(\mathrm{~m})$ & $1.86(\mathrm{~m})$ & 17 & $1.44(\mathrm{~m})$ & $1.46(\mathrm{~m})$ \\
2 & $1.76(\mathrm{~m})$ & $1.75(\mathrm{~m})$ & 18 & $0.73(\mathrm{~s})$ & $0.73(\mathrm{~s})$ \\
& $1.67(\mathrm{~m})$ & $1.66(\mathrm{~m})$ & 19 & $1.06(\mathrm{~s})$ & $1.05(\mathrm{~s})$ \\
$3 \alpha$ & $3.29(\mathrm{dd}, 4.6,11.6)$ & $3.29(\mathrm{dd}, 4.6,11.6)$ & 20 & $1.41(\mathrm{~m})$ & $1.43(\mathrm{~m})$ \\
$5 \alpha$ & $1.67(\mathrm{~m})$ & $1.66(\mathrm{~m})$ & 21 & $0.88(\mathrm{~d}, 6.0)$ & $0.93(\mathrm{~d}, 6.1)$ \\
$6 \alpha$ & $2.40(\mathrm{~m})$ & $2.41(\mathrm{~m})$ & 22 & $1.13(\mathrm{~m}), 1.55(\mathrm{~m})$ & $1.05(\mathrm{~m}), 1.48(\mathrm{~m})$ \\
$6 \beta$ & $2.38(\mathrm{~m})$ & $2.38(\mathrm{~m})$ & 23 & $1.90(\mathrm{~m}), 2.04(\mathrm{~m})$ & $1.82(\mathrm{~m}), 2.04(\mathrm{~m})$ \\
$11 \alpha$ & $2.37(\mathrm{~m})$ & $2.36(\mathrm{~m})$ & 24 & $5.09(\mathrm{~m})$ & $5.10(\mathrm{~m})$ \\
$11 \beta$ & $2.24(\mathrm{~m})$ & $2.24(\mathrm{~m})$ & 26 & $1.69(\mathrm{~s})$ & $1.68(\mathrm{~s})$ \\
$12 \alpha$ & $1.76-1.80(\mathrm{~m})$ & $1.76-1.80(\mathrm{~m})$ & 27 & $1.61(\mathrm{~s})$ & $1.60(\mathrm{~s})$ \\
$15 \alpha$ & $1.55(\mathrm{~m})$ & $1.55(\mathrm{~m})$ & 28 & $0.99(\mathrm{~s})$ & $0.99(\mathrm{~s})$ \\
$15 \beta$ & $2.12(\mathrm{~m})$ & $2.12(\mathrm{~m})$ & 29 & $0.88(\mathrm{~s})$ & $0.88(\mathrm{~s})$ \\
$16 \alpha$ & $1.34(\mathrm{~m})$ & $1.34(\mathrm{~m})$ & 30 & $0.97(\mathrm{~s})$ & $0.96(\mathrm{~s})$ \\
\hline
\end{tabular}

Epi-kansenone (11): colorless gum; $R_{\mathrm{f}}=0.64\left(\mathrm{SiO}_{2}\right.$, petroleum ether-chloroform-EtOAc $\left.=5: 3: 2\right)$; $\mathrm{UV}(\mathrm{MeOH}) \lambda_{\max } 252$; ESI-MS (positive) $m / z 441[\mathrm{M}+\mathrm{H}]^{+} ;{ }^{1} \mathrm{H}-\mathrm{NMR}$ data, see Table 3.

Euphol (12): white crystal (ethyl acetate); $\mathrm{mp} 116-118{ }^{\circ} \mathrm{C} ; R_{\mathrm{f}}=0.52\left(\mathrm{SiO}_{2}\right.$, hexane-acetone- glacial acetic acid $=8: 1: 0.1) ; \mathrm{UV}(\mathrm{MeOH}) \lambda_{\max } 200 ;{ }^{1} \mathrm{H}-\mathrm{NMR}\left(300 \mathrm{MHz}, \mathrm{CDCl}_{3}\right) \delta 0.75(3 \mathrm{H}, \mathrm{s}, 30-\mathrm{H}), 0.80$ $(3 \mathrm{H}, \mathrm{s}, 18-\mathrm{H}), 0.84(3 \mathrm{H}, \mathrm{d} J=3.3 \mathrm{~Hz}, 21-\mathrm{H}), 0.87$ (3H, s, 28-H), 0.95 (3H, s, 19-H), 1.00 (3H, s, 29-H), $1.60(3 \mathrm{H}, \mathrm{s}, 27-\mathrm{H}), 1.68(3 \mathrm{H}, \mathrm{s}, 26-\mathrm{H}), 3.24(1 \mathrm{H}, \mathrm{dd} J=4.8,11.6 \mathrm{~Hz}, 3 \alpha-\mathrm{H}), 5.09(1 \mathrm{H}, \mathrm{t}, J=7.5 \mathrm{~Hz}$, 24-H); ${ }^{13} \mathrm{C}-N M R\left(75 \mathrm{MHz}, \mathrm{CDCl}_{3}\right)$ : 35.4 (C-1), 27.7 (C-2), 78.9 (C-3), 37.3 (C-4), 50.9 (C-5), 18.9 (C-6), 27.9 (C-7), 134.0 (C-8), 133.5 (C-9), 38.9 (C-10), 21.5 (C-11), 28.1 (C-12), 44.1 (C-13), 49.9 (C-14), 30.9 (C-15), 29.8 (C-16), 49.6 (C-17), 15.6 (C-18), 20.1 (C-19), 35.8 (C-20), 18.9 (C-21), 35.2 (C-22), 24.7 (C-23), 125.2 (C-24), 130.8 (C-25), 25.7 (C-26), 17.7 (C-27), 24.4 (C-28), 28.0 (C-29), 15.5 (C-30).

\subsection{Cytotoxicity Activity on Human Normal Cell Lines of Extracts and Compounds from Kansui}

As shown in Table 4, the EtOH and EtOAc extract exhibited significant inhibition of cell proliferation against two human normal cell lines L-O2 and GES-1 with dose-dependent relationship. The $\mathrm{IC}_{50}$ values of EtOH extract on L-O2 and GES-1 were 42.02 and $30.67 \mu \mathrm{g} / \mathrm{mL}$, respectively. And the cytotoxicity of EtOAc extract on L-O2 and GES-1 were stronger than that of EtOH extract. The $\mathrm{IC}_{50}$ values of EtOAc extract on L-O2 and GES-1 were 27.08 and $21.89 \mu \mathrm{g} / \mathrm{mL}$, respectively. The water extract showed no obvious cytotoxicity against these two human normal cell lines. 
Table 4. The proliferation inhibition activities of kansui extract and compounds 1-12 on human normal liver cells L-O2 and gastric epithelial cell GES-1 $(n=6)$.

\begin{tabular}{ccc}
\hline \multirow{2}{*}{ Extract/Compounds } & \multicolumn{2}{c}{ IC $_{\mathbf{5 0}}$} \\
\cline { 2 - 3 } & L-O2 & GES-1 \\
EtOH extract & $42.02 \mu \mathrm{g} / \mathrm{mL}$ & $30.67 \mu \mathrm{g} / \mathrm{mL}$ \\
EtOAc extract & $27.08 \mu \mathrm{g} / \mathrm{mL}$ & $21.89 \mu \mathrm{g} / \mathrm{mL}$ \\
Water extract & $>100 \mu \mathrm{g} / \mathrm{mL}$ & $>100 \mu \mathrm{g} / \mathrm{mL}$ \\
1 & $>100 \mu \mathrm{M}$ & $>100 \mu \mathrm{M}$ \\
2 & $>100 \mu \mathrm{M}$ & $>100 \mu \mathrm{M}$ \\
3 & $>100 \mu \mathrm{M}$ & $>100 \mu \mathrm{M}$ \\
4 & $>100 \mu \mathrm{M}$ & $>100 \mu \mathrm{M}$ \\
5 & $12.40 \mu \mathrm{M}$ & $8.51 \mu \mathrm{M}$ \\
6 & $8.22 \mu \mathrm{M}$ & $6.67 \mu \mathrm{M}$ \\
7 & $13.08 \mu \mathrm{M}$ & $3.53 \mu \mathrm{M}$ \\
8 & $70.78 \mu \mathrm{M}$ & $23.51 \mu \mathrm{M}$ \\
9 & $34.53 \mu \mathrm{M}$ & $22.01 \mu \mathrm{M}$ \\
10 & $14.36 \mu \mathrm{M}$ & $13.44 \mu \mathrm{M}$ \\
11 & $17.82 \mu \mathrm{M}$ & $8.04 \mu \mathrm{M}$ \\
12 & $>100 \mu \mathrm{M}$ & $>100 \mu \mathrm{M}$ \\
\hline
\end{tabular}

Figure 2. The cytotoxicity and dose-dependent relationship of compounds 5-11 on human normal cell lines GES-1.

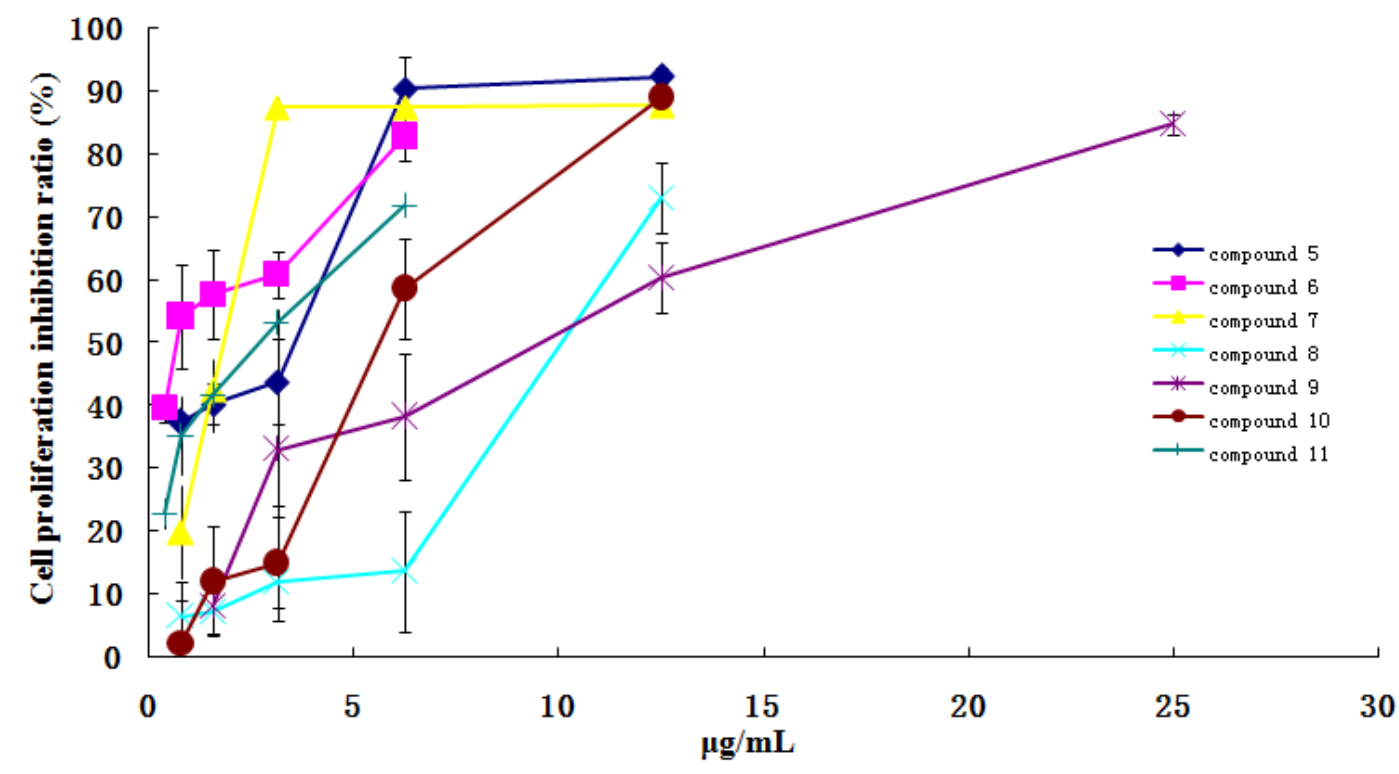

Compared with the control group, compounds 5, 7, 8 and 10 in the concentration range of $0.78-12.5 \mu \mathrm{g} / \mathrm{mL}$, compounds 6 and 11 in the concentration range of $0.39-6.25 \mu \mathrm{g} / \mathrm{mL}$, and compound 9 in the concentration range of 1.56-25 $\mu \mathrm{g} / \mathrm{mL}$, showed significant inhibition activity on GES-1 cell lines growth with a dose-dependent relationship (Figure 2). And compounds 5, 6, 7, 10 and 11 in the concentration range of $0.78-12.5 \mu \mathrm{g} / \mathrm{mL}$, compound 9 in the concentration range of $1.56-25 \mu \mathrm{g} / \mathrm{mL}$, and compound 8 in the concentration range of $3.125-50 \mu \mathrm{g} / \mathrm{mL}$, had significant inhibition activity on L-O2 cell lines growth with a dose-dependent relationship (Figure 3). Whereas, 
other compounds did not show the obvious cytotoxicity activity in the concentration range of 3.125-50 $\mu \mathrm{g} / \mathrm{mL}$. Considering their structure, the results indicated that ingenane-type diterpenoids (5-9) except compound 4 and 8-ene-7-one triterpenoids (10 and 11) in kansui possessed stronger gastrointestinal toxicity and hepatotoxicity than other compounds. More interestingly, the $\mathrm{IC}_{50}$ values of 5-11 and the kansui extracts on GES-1 cell lines were less than those on L-O2 cell lines, which suggested that the gastrointestinal toxicity of kansui is stronger than its hepatotoxicity. This result coincided with the clinical toxic performance of kansui [14-16].

Figure 3. The cytotoxicity and dose-dependent relationship of compounds 5-11 on human normal cell lines L-O2.

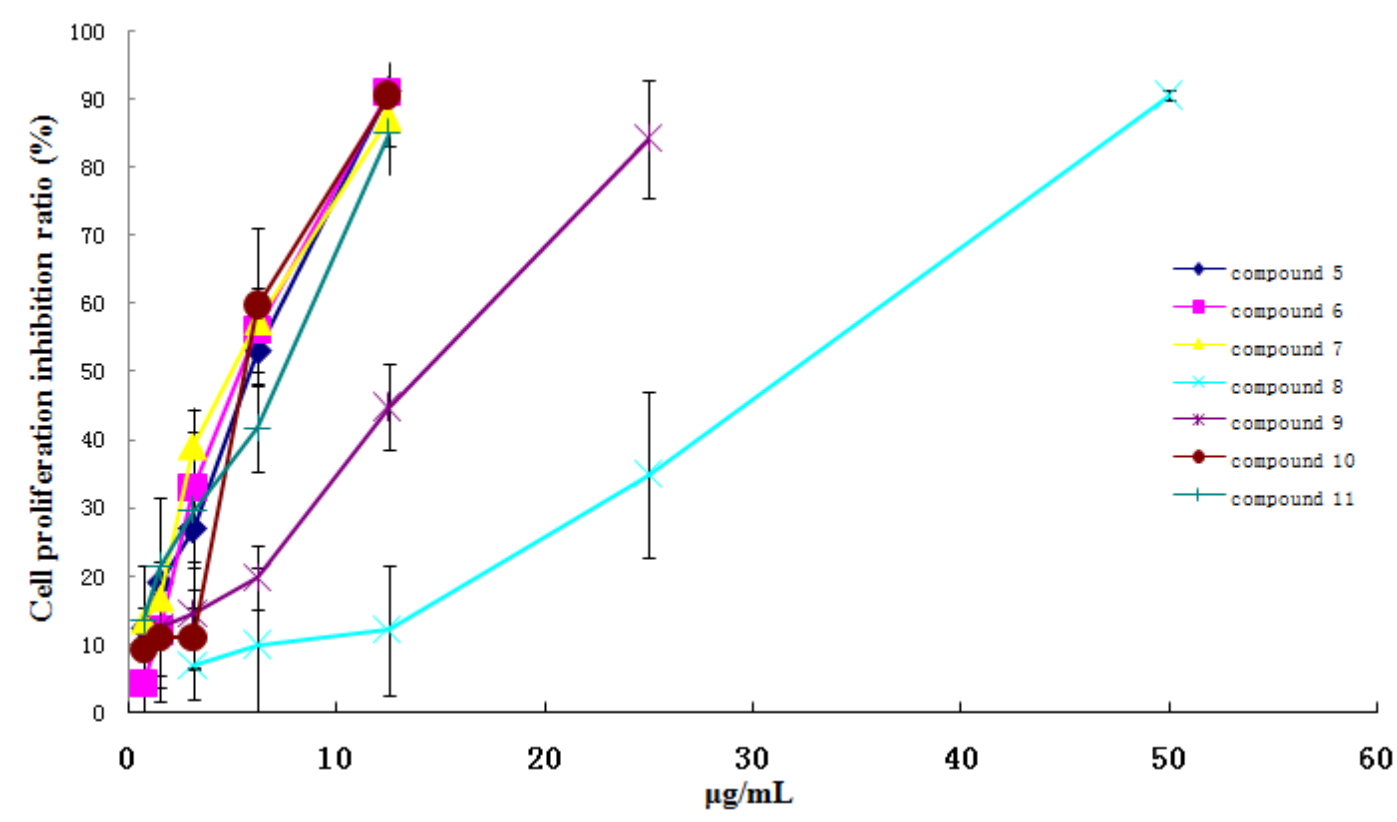

\section{Experimental Section}

\subsection{Chemicals and Reagents}

Analytical grade ethanol, EtOAc and petroleum ether $\left(60-90{ }^{\circ} \mathrm{C}\right)$ (Sinopharm Chemical Reagent Co., Ltd. Shanghai, China) were used for extraction and isolation. The silica gel (Qingdao Ocean chemical industry, Qingdao, China) was used for column chromatography. Dulbecco's Modified Eagle's Medium (DMEM) was purchased from Gibco Co., Ltd. (Grand Island, NY, USA); fetal bovine serum (FBS) and calf serum (CS) were purchased from Sijiqing Biological Engineering Material Co., Ltd. (Hangzhou, China); 3-(4,5-dimethylthiazol-2-yl)-2,5-diphenyltetrazolium bromide (MTT) was obtained from Beijing Solarbio science and technology Co., Ltd. (Beijing, China); PBS buffer was purchased from Boster Bio-engineering Co., Ltd. (Wuhan, China); dimethylsulfoxide (DMSO) was purchased from Sinopharm Chemical Reagent Co., Ltd. (A·R grade, Shanghai, China). 


\subsection{Instrumentation}

Melting points were determined on a Yanagimoto MP-500 D micro-melting point apparatus and are uncorrected. The UV spectrum was obtained in $\mathrm{MeOH}$ on a Shimadzu UV-2401 spectrophotometer. The NMR spectra were recorded on Bruker Avance AV 300 instrument operating at $300 \mathrm{MHz}$ for ${ }^{1} \mathrm{H}$ and $75 \mathrm{MHz}$ for ${ }^{13} \mathrm{C}$, using standard pulse sequences. Chemical shifts are reported on the $\delta$ scale in parts per million, with TMS as an internal standard. The electrospray ionization mass spectra (ESI-MS) were obtained on a Q-TOF Micro mass spectrometer (Waters Company, Milford, MA, USA). Thin-layer chromatography (TLC) was performed on Qingdao Ocean TLC plates $(0.25 \mathrm{~mm}$ thickness, Qingdao Ocean chemical industry, Qingdao, China), with compounds visualized by spraying with $10 \%(v / v) \mathrm{H}_{2} \mathrm{SO}_{4}$ in ethanol solution and then heating on a hot plate. Semipreparative HPLC was performed on a Hanbang NP 7000 apparatus with a NU 3000 detector. A Hanbang Phecda Si silica gel column $(20 \times 250 \mathrm{~mm}$ i.d., $5 \mu \mathrm{m})$ was used for preparative purposes. Laminar flow clean bench (Model: SW-CJ-1F) was purchased from Suzhou Purification Equipment Co., Ltd. (Suzhou, China); $\mathrm{CO}_{2}$ incubator (Model: MCO-20AIC) was purchased from Sanyo Denki Co., Ltd. (Moriguchi, Osaka, Japan); Microplate Reader (model: 680) obtained from Bio-Rad Laboratories (Hercules, CA, USA). Inverted phase contrast microscope (model: CKX31) was purchased from Olympus Co., Ltd. (Tokyo, Japan).

\subsection{Plant Material}

The roots of Euphorbia kansui T.N. Liou ex T.P. Wang were collected from Red River valley of Baoji (Shanxi Province, China). The crude plant was identified by Professor Chungen Wang (Nanjing University of Chinese Medicine, Nanjing, China). The voucher specimens (No. NJUTCM-20091008) was deposited in the Herbarium of Nanjing University of Chinese Medicine, Jiangsu, China.

\subsection{Extraction and Isolation}

The dried and crushed roots of E. kansui $(20.0 \mathrm{~kg})$ were extracted with $95 \% \mathrm{EtOH}$ under $50{ }^{\circ} \mathrm{C}$ water bath, the supernatant was separated every day, and the solvent was removed under reduced pressure, and the residue $(2.35 \mathrm{~kg})$ was partitioned with ethyl acetate and water to provide the EtOAc fraction $(1.032 \mathrm{~kg})$ and water fraction $(1.056 \mathrm{~kg})$. The EtOAc fraction $0.904 \mathrm{~kg}$ was subjected to silica gel column chromatography $(100 \times 10 \mathrm{~cm}, 200-300$ mesh, eluted with petroleum ether and ethyl acetate in increasing polarity). The column chromatographic eluents (500 mL each) were concentrated under reduced pressure and combined according to TLC monitoring into 10 fractions. Fraction 1 was subjected to silica gel column chromatography eluted with petroleum ether-ethyl acetate 100:1, and was further recrystallized to give compound 12 (a large amount). Fractions 2-4 was subjected to silica gel column chromatography eluted with petroleum ether-ethyl acetate from 100:16 to 100:18, and were further recrystallized to give $\mathbf{1}(132 \mathrm{mg}), \mathbf{2}(86 \mathrm{mg})$ and $\mathbf{3}(46 \mathrm{mg})$. Fraction 5 was subjected to silica gel column chromatography eluted with petroleum ether-ethyl acetate 100:2, and was further purified by preparative TLC using silica gel (petroleum ether-chloroform-ethyl acetate 5:3:1, $R_{\mathrm{f}}=0.83$ ) to give compound 4 (106 mg). Fraction 6 was subjected to silica gel column chromatography eluted with petroleum ether-ethyl acetate 100:5, and was further purified by preparative HPLC (petroleum 
ether-ethyl acetate, $\rho 0.705$, UV detector set at $260 \mathrm{~nm}$, flow rate at $15 \mathrm{~mL} / \mathrm{min}$ ) to give compound 9 (41 $\mathrm{mg}, t_{\mathrm{R}} 31.0 \mathrm{~min}$ ). Fraction 7 was subjected to silica gel column chromatography eluted with petroleum ether-ethyl acetate 100:5, and was further purified by preparative HPLC (petroleum ether-ethyl acetate, $\rho 0.700$, UV detector set at $260 \mathrm{~nm}$, flow rate at $15 \mathrm{~mL} / \mathrm{min}$ ) to give compound 7 (56 $\mathrm{mg}, t_{\mathrm{R}} 14.3 \mathrm{~min}$ ). Fraction 8 was subjected to silica gel column chromatography eluted with petroleum ether-ethyl acetate 100:6, and was further purified by preparative HPLC (petroleum ether-ethyl acetate, $\rho 0.692$, UV detector set at $260 \mathrm{~nm}$, flow rate at $15 \mathrm{~mL} / \mathrm{min}$ ) to give compound 8 (14 $\mathrm{mg}, t_{\mathrm{R}} 16.4 \mathrm{~min}$ ). Fraction 9 was subjected to silica gel column chromatography eluted with petroleum ether-ethyl acetate 100:8, and was further purified by preparative HPLC (petroleum ether-ethyl acetate , $\rho 0.706$, UV detector set at $260 \mathrm{~nm}$, flow rate at $15 \mathrm{~mL} / \mathrm{min}$ ) to give compound 10 $\left(73 \mathrm{mg}, t_{\mathrm{R}} 20.6 \mathrm{~min}\right)$ and $11\left(32 \mathrm{mg}, t_{\mathrm{R}} 22.7 \mathrm{~min}\right)$. Fraction 10 was subjected to silica gel column chromatography eluted with petroleum ether-ethyl acetate 100:11, and was further purified by preparative HPLC (petroleum ether-ethyl acetate , $\rho 0.705$, UV detector set at $260 \mathrm{~nm}$, flow rate at $15 \mathrm{~mL} / \mathrm{min})$ to give compound $5\left(29 \mathrm{mg}, t_{\mathrm{R}} 19.0 \mathrm{~min}\right)$ and $6\left(21 \mathrm{mg}, t_{\mathrm{R}} 20.3 \mathrm{~min}\right)$.

\subsection{Cell Line and Cell Culture}

Human normal liver cell L-O2 was kindly donated by the School of Life Science, Nanjing Normal University (Nanjing, China), and Human normal gastric epithelial cell GES-1 was kindly donated by the Department of Gastroenterology, Nanjing Drum Tower Hospital (Nanjing, China). Human normal liver cell LO-2 was routinely cultured in DMEM medium [16], supplemented with $10 \%(v / v)$ fetal bovine serum (FBS), $100 \mathrm{U} / \mathrm{mL}$ of penicillin and $100 \mu \mathrm{g} / \mathrm{mL}$ of streptomycin in a humidified atmosphere with $5 \% \mathrm{CO}_{2}$ at $37{ }^{\circ} \mathrm{C}$ in an incubator. The culture medium was changed every day. Cultures were dissociated with $0.25 \%$ trypsase in phosphate buffered ( $\mathrm{pH} 7.2 \sim 7.4$ ) saline (PBS). Human normal gastric epithelial cell GES-1 was routinely cultured in DMEM medium [30], supplemented with $15 \%(v / v)$ calf serum (CS), $100 \mathrm{U} / \mathrm{mL}$ of penicillin and $100 \mu \mathrm{g} / \mathrm{mL}$ of streptomycin in a humidified atmosphere with $5 \% \mathrm{CO}_{2}$ at $37{ }^{\circ} \mathrm{C}$ in an incubator. The culture medium was changed every two days. Cultures were dissociated with $0.25 \%$ trypsase and $0.05 \%$ EDTA in PBS (pH 7.2 7.4).

\subsection{Cell Proliferation Analysis}

Cytotoxicity activity was assayed by MTT method as described [31]. Briefly, cells were seeded in 96-well plates (Costar, Corning Co., Ltd., Beijing, China) with $100 \mu \mathrm{L}$ at a concentration of cell suspension of $1.0 \times 10^{4}$ cells $/ \mathrm{mL}$ (L-O2 cell) and $1.0 \times 10^{5}$ cells $/ \mathrm{mL}$ (GES-1 cell), and incubated for $24 \mathrm{~h}$ at $37{ }^{\circ} \mathrm{C}$ in a humidified atmosphere of $5 \% \mathrm{CO}_{2}$ in an incubator.

Every sample was solubilized in dimethyl sulfoxide (DMSO) to a concentration of $10 \mathrm{mg} / \mathrm{mL}$ and stored at $4{ }^{\circ} \mathrm{C}$, then diluted with complete medium to the desired series concentration before treating the cells. Each solution was added into 96-well plates with $100 \mu \mathrm{L}$ per well, repeated 6 well. Cells of the control groups were treated with the same volume of medium. The treated cells with different groups were incubated for $48 \mathrm{~h}$ at $37^{\circ} \mathrm{C}$ in a humidified atmosphere of $5 \% \mathrm{CO}_{2}$ in an incubator. Then, the treated cells were added with $20 \mu \mathrm{L} /$ well of MTT $(5.0 \mathrm{mg} / \mathrm{mL})$, and incubated for a further $4 \mathrm{~h}$ in an incubator. The growth medium was removed from all the wells, and $150 \mu \mathrm{L}$ DMSO were added to 
each well. The plates were shaken gently for $10 \mathrm{~min}$ to blend the mixture. The absorbance value of each well was read at $490 \mathrm{~nm}$ using a microplate reader. All experiments were performed at least three times. The inhibition rate of cell proliferation was calculated according to the formula:

cell inhibition $(\%)=(1-$ sample solution absorbance value/control absorbance value $) \times 100 \%$

\subsection{Statistical Analysis}

The results were expressed as an inhibition ratio and $\mathrm{IC}_{50}$ (concentration that inhibits $50 \%$ of cell growth). The data were analyzed with software SPSS 15.0 (SPSS Inc.: Chicago, IL, USA). The anti-proliferation activity is more significant when the $\mathrm{IC}_{50}$ is smaller.

\section{Conclusions}

In this paper, the 95\% EtOH extract of kansui exhibited significant activity against two human normal cell lines GES-1 and L-O2. By bio-guided isolation, 12 terpenoid compounds, including jatrophane-type diterpenoids (1-3) ingenane-type diterpenoids (4-9) and triterpenoids (10-12), were isolated and identified from the active fraction of kansui. The results showed that five ingenane-type diterpenoids (5-9) and two triterpenoids (10 and 11) exhibited relative lower $\mathrm{IC}_{50}$ value and thus, stronger potential cytotoxic activity against different human normal cell lines in a dose-dependent fashion. The ingenane-type diterpenoids with 3-unsaturated aliphatic chain, such as compounds 5, 6 and 7, possessed stronger cytotoxic activity. And the cytotoxic activity was slightly decreased if the 3-unsaturated aliphatic chain was replaced by other groups, such as methyl and phenyl groups; and there was the same decrease of cytotoxic activity if the 5-hydroxyl group was replaced by a benzoyl group. And 20-O group had little influence on the cytotoxic activity. The three jatrophane-type diterpenoids (1-3) showed very weak cytotoxic activity against the two human normal cell lines. The triterpenoids with 8-ene-7-one showed stronger cytotoxic activity. For example, compound 10 (kansenone) and $\mathbf{1 1}$ (Epi-kansenone) had stronger cytotoxic activity than that of compound $\mathbf{1 2}$ (euphol). It is also notable that all identified toxic terpenoids were also reported in the literature to be important pharmaceutical ingredients for anti-tumor, anti-allergy, anti-virus, and antinematodal activity [2-5,13,17-19]. Therefore, it is essential that the dose of kansui should be controlled within an appropriate range in the clinical application to assure its safe and efficacy.

\section{Acknowledgments}

This research was financially supported by the National Natural Science Foundation of China (30973940), the National Basic Research Program of China ("973 Program”) (2011CB505300, 2011CB505303), the 2009' Program for Excellent Scientific and Technological Innovation Team of Jiangsu Higher Education, and the 2009' Program for New Century Excellent Talents by the Ministry of Education (NCET-09-0163). This research was also supported by a Project Funded by the Priority Academic Program Development of Jiangsu Higher Education Institutions (ysxk-2010), Jiangsu Government Scholarship for Overseas Studies (JS-2009-061), and Jiangsu Provincial Talents in Six Fields (2010). In addition, this work was completed in Class III Laboratory of Chemistry of Chinese Materia Medica of State Administration of Traditional Chinese Medicine of China. 


\section{References}

1. Chinese Pharmacopoeia Commission. Pharmacopoeia of the People's Republic of China, 9th ed.; China Medical Science Press: Beijing, China, 2010; Volume I , p. 241.

2. Wu, T.S.; Lin, Y.M.; Haruna, W.; Pan, D.J.; Shingu, T. Kansuiphorbia A and B, two novel antileukemic deterpene ester from Euphorbia kansui. J. Nat. Prod. 1991, 54, 823-829.

3. Miyata, S.; Wang, Y.L.; Yoshida, C. Inhibition of cellular proliferation by diterpenes, topoisomerase II inhibitor. Bioorg. Med. Chem. 2006, 14, 2048-2051.

4. Wang, L.Y.; Wang, N.L.; Yao, X.S.; Miyata, S.; Kitanaka, S. Diterpenes from the roots of Euphorbia kansui and their in vitro effects on the cell division of Xenopus (2). Chem. Pharm. Bull. 2003, 51, 935-941.

5. Wang, L.Y.; Wang, N.L.; Yao, X.S.; Miyata, S.; Kitanaka, S. Diterpenes from the roots of Euphorbia kansui and their in vitro effects on the cell division of Xenopus. J. Nat. Prod. 2002, 65, 1246-1251.

6. Yu, F.R.; Lian, X.Z.; Guo, H.Y.; McGuire, P.M.; Li, R.D.; Wang, R.; Yu, F.H. Isolation and characterization of methyl esters and derivatives from Euphorbia kansui (Euphorbiaceae) and their inhibitory effects on the human SGC-7901 cells. J. Pharm. Pharmaceut. Sci. 2005, 8, 528-535.

7. Li, X.R.; Zhang, Y.D.; Tang, H.H.; Wu, F.Y. Study of auxiliary therapeutic effect of kansui root on patients with severe acute pancreatitis (in Chinese). China J. Modern Med. 2002, 12, 7-9.

8. Ouyang, J.B.; Deng, M.Y.; Ouyang, Y.M. Therapeutic effect on adjuvant treatment of severe acute pancreatitis with Euphorbia kansui Lious (in Chinese). China J. Modern Med. 2004, 14, 96-97.

9. Lu, X.S.; Zhang, Y.; Ai, Y.H.; Li, Y.X. The clinical study of kansui root therapy for severe acute pancereatitis (in Chinese). J. Chin. Phys. 2004, 6, 1444-1447.

10. Fan, Y.; Cai, D.F.; Gu, X.X.; Wang, G.H.; Ma, J. Treatment of intestinal obstruction with a large dose of kansui (in Chinese). J. Emerg. Tradit. Chin. Med. 2005, 14, 278-279.

11. Liu, J.; Hong, L.J.; Guang, L.H.; Feng, W.L.; Bo, X.D.; Xian, M.F.; Ying, L.; Ning, Z. Treatment of postoperative intestinal obstruction in 504 cases with kansui (in Chinese). Chin. J. Critic. Care Med. 1998, 18,45 .

12. Matsumoto, T.; Cyong, J.C.; Yamada, H. Stimulatory effects of ingenols from Euphorbia kansui on the expression of macrophage Fc receptor. Planta. Med. 1992, 58, 255-258.

13. Zheng, W.F.; Cui, Z.; Zhu, Q. Cytotoxicity and antiviral activity of the compounds from Euphorbia kansui. Planta. Med. 1998, 64, 754-756.

14. Huang, H.Y.; Shu, X.Y.; Ding, A.W.; Li, Z. Experimental study on purgative activity and acute toxicity of ethanol extracts from different polar parts of kansui and vinegar-preparing kansui (in Chinese). China Pharm.2008, 17, 3-4.

15. Xiang, L.H.; Chen, Y.P.; Zhang, Z.; Shan, Z.Y.; Yu, Z.M.; Lv, A.P. The effect of 24 kinds toxic chinese medicine long-term toxicity experiments on rat organ index (in Chinese). Chin. J. Basic Med. Tradit. Chin. Med. 2006, 12, 35-36, 52.

16. Yan, X.J.; Zhang, L.; Li, L.; Cao, Y.D.; Li, Z.J.; Tang, Y.P.; Ding, A.W. Study on detoxication of kansui radix on normal liver cells LO2 after stir-baking with vinegar (in Chinese). China J. Chin. Materia. Medica. 2012, 37, 1667-1671. 
17. Wang, L.Y. Study on chemical constituents and biological activity of kansui. Ph.D. Thesis, Shenyang Pharmaceutical University, Shenyang, China, July, 2003.

18. Pan, Q.; Ip, F.C.; Ip, N.Y.; Zhu, H.X.; Min, Z.D. Activity of macrocyclic jatrophane diterpenes from Euphorbia kansui in a TrkA fibroblast survival assay. J. Nat. Prod. 2004, 67, 1548-1551.

19. Pan, Q.; Min, Z.D. Studies on ingenol-type diterpene esters in root tuber of Euphorbia kansui (in Chinese). Chin. Tradit. Herb Drugs 2003, 34, 489-492.

20. Pan, D.J.; Qi, H.C.; Chang, J.J.; Lee, T.T.Y.; Chen, Y.P.; Hsu, H.Y.; McPhail, D.R.; McPhail, A.T.; Lee, K.H. Kansuiphorin C and D, cytotoxic diterpenes from Euphorbia kansui. Phytochemistry 1991, 30, 1018-1020.

21. Li, C.F. Studies of the constituents of the processed roots of Euphorbia kansui T.N.Wang. Master's Thesis, Shenyang Pharmaceutical University, Shenyang, China, May, 2006.

22. Uemura, D.; Ohwaki, H.; Hirata, Y. Isolation and structures of 20-deoxyingenol, new diterpene, derivatives, and ingenol derivative obtained from kansui. Tetrahedron Lett. 1974, 29, 2527-2528.

23. Wang, Y.B.; Li, Y.Y.; Wang, H.B.; Qin, G.W. Chemical constituents from the roots of Euphorbia kansui. Chin. J. Nat. Med. 2007, 5, 182-185.

24. Nunomura, S.; Kitanaka, S.; Ra, C. 3-O-(2,3-Dimethylbutanoyl)-13-O-decanoylingenol from Euphorbia kansui Suppresses IgE-Mediated Mast Cell Activation. Biol. Pharm. Bull. 2006, 29, 286-290.

25. Zeng, Y.; Zhong, J.M.; Ye, S.Q.; Ni, Z.Y.; Miao, X.Q.; Mo, Y.K.; Li, Z.L. Screening of Epstein-Barr virus early antigen expression inducers from Chinese medicinal herbs and plants. Biomed. Environ. Sci. 1994, 7, 50-55.

26. Shi, J.X.; Li, Z.X.; Nitoda, T.; Izumi, M.; Kanzaki, H.; Baba, N.; Kawazu, K.; Nakajima, S. Three antinematodal diterpenes from Euphorbia kansui. Biosci. Biotechnol. Biochem. 2007, 71, 1086-1089.

27. Geng, T.; Huang, H.Y.; Ding, A.W.; Zhang, L. Irritation and diarrhea effect of different polar parts of Euphorbia kansui T. and vinegar preparing Euphorbia kansui T (in Chinese). Cent. South Pharm. 2008, 6, 385-388.

28. Shu, X.Y.; Yu, L.; Tang, Y.P.; Zhang, L.; Ding, A.W.; Duan, J.A.; Shen, X.C. Bioassay-guided separation of the proinflammatory constituents from the roots of Euphorbia kansui. J. Nat. Med. 2010, 64, 98-103.

29. Zhang, L.; Shu, X.Y.; Ding, A.W.; Yu, L.; Tang, Y.P.; Duan, J.A.; Shang, E.X.; Shen, X.C. LC-DAD-ESI-MS-MS Separation and chemical characterization of the inflammatory fraction of the roots of Euphorbia kansui. Chromatographia 2009, 70, 805-810.

30. Wang, Y.; Liu, J.; Cui, J.F.; Xing, L.X.; Wang, J.L.; Yan, X.; Zhang, X.H. ERK and p38 MAPK signaling pathways are involved in ochratoxin A-induced G2 phase arrest in human gastric epithelium cells. Toxicol. Lett. 2012, 209, 186-192.

31. Yarim, M.; Koksal, M.; Durmaz, I.; Atalay, R. Cancer cell cytotoxicities of 1-(4-substitutedbenzoyl)-4-(4-chlorobenzhydryl)piperazine derivatives. Int. J. Mol. Sci. 2012, 13, 8071-8085.

(C) 2012 by the authors; licensee MDPI, Basel, Switzerland. This article is an open access article distributed under the terms and conditions of the Creative Commons Attribution license (http://creativecommons.org/licenses/by/3.0/). 